\title{
THE PRELIMINARY MEETING OF THE SOUTHWESTERN SECTION.
}

THE preliminary meeting of the proposed Southwestern Section* of the American Mathematical Societry took place at Columbia, Mo., on the 1st of December, 1906. About thirty-five persons, including the following members of the Society, were present :

Professor L. D. Ames, Mr. R. L. Börger, Professor A. S. Chessin, Brother Constantius, Professor G. R. Dean, Dr. E. L. Dodd, Dr. F. J. Dohmen, Mr. E. S. Haynes, Professor E. R. Hedrick, Professor O. D. Kellogg, Mr. W. A. Luby, Professor E. H. Moore, Professor Alexander Pell, Professor Oscar Schmiedel, Mr. F. C. Touton, Miss M. S. Walker, Dr. Paul Wernicke, Dr. W. D. A. Westfall, Mr, A. M. Wilson, Professor J. W. Withers.

The meeting was called to order by Professor Hedrick at 10:30 A. M. Professor Hedrick was elected chairman and Professor Chessin secretary of the meeting. Professor E. H. Moore was asked to preside as honorary chairman of the meeting for the afternoon session. The morning session adjourned at $1 \mathrm{P} . \mathrm{M}$.

The meeting was called to order for the afternoon session at 2:30 P. M. The reading of papers was preceded by a short business meeting. On the motion made by Professor Chessin the members present unanimously selected St. Louis as the next meeting place of the proposed Southwestern Section.

A programme committee was elected, consisting of Professor E. R. Hedrick, chairman ; Professor A. S. Chessin, secretary ; Professor M. B. Porter.

At the conclusion of the meeting a motion was passed expressing the thanks of the members to all who had assisted in the formation of this section, especially to Professor E. H. Moore. The meeting adjourned at 5:45 P. M., and was followed by an informal supper at which about twenty were present.

* The formation of the Southwestern Section was authorized at the meeting of the Council on December 28. 
The following papers were read:

(1) Professor L. D. Ames: "Notes on the orientation of a secant."

(2) Mr. R. L. BöRGER : "On the determination of the subgroups of the special linear ternary homogeneous group in the Galois field $p^{2}$ " (preliminary report).

(3) Professor A. S. Chessin : "On a particular case of motion of Sire's polytrope and Foucault's gyroscope."

(4) Professor G. R. Dean : "The equations of motion of a compressible frictionless fluid" (preliminary report).

(5) Dr. E. L. DodD : "Incomplete double sequences as exemplified by infinitesimals."

(6) Professor S. Epsteen: "The probable error of a measurement a unit in length."

(7) Dr. O. E. GleNN : "On a class of operation groups of order $p_{1}^{m_{1} \mu_{1}} p_{2}{ }^{m_{2} \mu_{2}}$."

(8) Professor O. D. KelloGG: "Harmonic functions on the boundary of their region of definition."

(9) Professor Oscar Schmiedel : "The polynomial coefficient generalized and extended."

(10) Professor E. H. Moore : "Homogeneous distributive functional operations of degree $n . "$

(11) Professor H. B. Newson : "Real conformal transformations in space."

(12) Professor G. A. Miller: "Groups in which every subgroup is either abelian or dihedral."

(13) Professor M. B. Ponter: "Change of variable in a multiple integral."

(14) Professor M. B. Porter : "Pringsheim's criterion for expansibility in Taylor's series."

(15) Dr. Paul Wernicke : "On the interlocking and knotted curves and surfaces."

(16) Dr. W. D. A. Westfalle: "Generalization of the fundamental theorem of Fourier constants."

(17) Professor E. H. Moore : "Notes on Fourier's constants."

(18) Professor L. E. Dickson : "Invariants of binary forms under modular transformations."

(19) Professor Alexander Pell: "Some remarks on curves of constant torsion."

(20) Professor M. B. Porter : " Polynomial convergents of a power series." 
In the absence of the authors Dr. Glenn's paper was read by Professor Kellogg, Professor Dickson's by Professor Hedrick, Professor Porter's third paper by Dr. Westfall, and Professor Miller's paper and Professor Porter's first two papers were read by title. Abstracts of the papers follow below. The abstracts are numbered to correspond to the titles in the list above.

1. In certain problems in the theory of potential it is desirable to be able to define uniquely the positive sense of a secant to a smooth curve, and also to define the angle it makes with a given line as a single valued continuous function of the points of intersection. Professor Ames showed that this is conveniently done by means of the definition of an angle from its sine and cosine taken together, as used in a previous paper (American Journal of Mathematics, October, 1905, page 349).

2. In Mr. Börger's paper the subgroups of order a power of $p$ and those of order a multiple of $p$ down to the order $p^{3} M(M$ being prime to $p$ ) are determined for the special linear ternary homogeneous groups in a Galois field $p^{2}$.

3. When the mass of the rings in the apparatus is neglected the motion of Sire's polytrope or Foucault's gyroscope is expressible by means of elliptic or circular functions. Professor Chessin showed that the solution could be also effected in terms of elliptic functions in the case when $A_{1}=A+C_{1} ; A_{1}, A_{1}, C_{1}$ and $A, A, C$ indicating respectively the principal moments of inertia of the internal ring and of the torus. Such a relation may be obtained by a proper construction of the gyroscope.

4. For the case of steady motion in two dimensions under no forces we have the equations

$$
u \frac{\partial u}{\partial x}+v \frac{\partial u}{\partial y}=-\frac{1}{\rho} \frac{\partial p}{\partial x}, \quad u \frac{\partial v}{\partial x}+v \frac{\partial v}{\partial y}=-\frac{1}{\rho} \frac{\partial p}{\partial y}
$$

and the equation of continuity

$$
\frac{\partial u}{\partial x}+\frac{\partial v}{\partial y}=-\frac{1}{\rho}\left(u \frac{\partial \rho}{\partial x}+v \frac{\partial \rho}{\partial y}\right)
$$


Eliminating $p$ and $\rho$ by means of the isothermal relation $p=k \rho$, Professor Dean obtains an equation of the second order of Monge's form which is easily integrated, and the arbitrary functions may be determined to fit given boundary conditions. The adiabatic relation also gives a Monge equation.

If the acceleration due to gravity is introduced, another term is introduced into the equation, rendering the subsidiary system of pfaffians inexact, $i$. e., not capable of being represented by a single integral equation.

In the case of motion in three dimensions, both the isother$\mathrm{mal}$ and adiabatic relations give equations in three independent variables analogous to Monge's. In the case of no forces the system of pfaffians is exact, but when forces are introduced the system does not satisfy the conditions of integrability.

5. Dr. Dodd notices that the totality of the infinitesimals $a_{i n}$, when $n \doteq \infty$, forms an incomplete double sequence, which lacks the terms on one side of the main diagonal. A common error in the statement of a theorem regarding the substitution of infinitesimals in an infinite sum may be made clear by two sequences whose terms are: $a_{i n}=1 / n$ and $\beta_{i n}=1 /(n+1-i)$ respectively. In this case,

$\lim _{n=\infty} \beta_{i n} / a_{i n}=1 ; \lim _{n=\infty} \sum_{i=1}^{i=n} a_{i n}=1 . \quad B u t \lim _{n=\infty} \sum_{i=1}^{i=n} \beta_{i n}=\infty$.

6. Basing his argument on an assumption which differs from the one used in Merriman's Least Squares, Professor Epsteen obtains a different value for the probable error of a measurement a unit in length, viz., $0.6745 \sqrt{\sum p d^{2}(2 n-1)^{-1}}$ where $p=2\left(l+l^{\prime}\right)^{-1}$, and $d=l-l^{\prime}, l$ and $l^{\prime}$ being duplicate measurements.

7. The first part of Dr. Glenn's paper was published in full in the Bulletin, May, 1906. In the second part he extends the methods there employed to include the determination of the defining relations of a class of groups of the given order, whose subgroup of order $p_{1}^{m_{1} \mu_{1}}$ is the abelian type $\left[\mu_{1}, \mu_{1}, \cdots, \mu_{1}\right]$, $\left(\mu_{1}>1\right)$. A general method of determining the number of distinct types in these relations is developed.

8. In this paper Professor Kellogg extends the investigations announced at the September meeting of the Society to 
regions other than the circle. An apparently new view point is attained in seeking conditions for the existence of the derivatives on the boundary which bear as nearly as possible equally upon boundary curve and boundary values. Restricting the problem to normal derivatives the following result is obtained : Let $x=x(s), y=y(s)$ be the parameter equations of the curve, $s$ being the length of arc between a fixed and a varying point, and let $f(s)$ be the boundary values to be approached by the potential function. Then if three positive constants $A, a$ and $\delta$ can be determined, such that for every $s\left|\Delta x^{\prime}(s)\right|<A|\Delta s|^{a}$ and $\left|\Delta y^{\prime}(s)\right|<A|\Delta s|^{a}$ as soon as $|\Delta s|<\delta$; and if the integral

$$
\int\left|\frac{f^{\prime}(s+t)-f^{\prime}(s)}{t}\right| d t
$$

is convergent for every value of $s$ when extended over an interval containing $t=0$ in its interior, then the derivative of the potential function formed in the direction of a normal to the boundary curve approaches a finite limit as the boundary is approached along the normal, and these limits form a continuous function on the boundary. Incidentally a generalization of Fredholm's proof of the Dirichlet principle is obtained, the boundary curve being subject only to the above restriction and to being a single closed curve without double points ; and some new light is thrown upon the potentials of double distributions on a curve.

9. The binomial coefficient represented in Grassmann's notation by $n^{\cdot a}$ is generalized by Professor Schmiedel to embrace negative values of $a$ and extended, the symbol $n^{a, b . c \ldots . r}$ being introduced. The fundamental relations of the extended coefficients are derived and numerous applications are shown, notably the forming of the $n$th derivative of a function of several variables.

10. In generalization of current concepts of linear distributive functional operations Professor Moore proposes the following definition :

On the supposition that $(a)$ the notations $u$ relate to elements of a field $F$ having no modulus; (b) the notations $\phi$ relate to elements of a class $\mathfrak{M}$ linear with respect to $F, i$. e., such that the notations 


$$
\begin{gathered}
u \phi=\phi u, \quad u_{1} \phi_{1}+u_{2} \phi_{2}=u_{2} \phi_{2}+u_{1} \phi_{1}, \quad 1 \phi=\phi, \quad 0 \phi=0, \\
\left(u_{1}+u_{2}\right) \phi=u_{1} \phi+u_{2} \phi, u\left(\phi_{1}+\phi_{2}\right)=u \phi_{1}+u \phi_{2}, \quad u_{1}\left(u_{2} \phi\right)=\left(u_{1} u_{2}\right) \phi
\end{gathered}
$$

specify in each case definite elements of $\mathfrak{M}$; and (c) the notations $\phi^{*}$ relate to elements of a class $\mathfrak{M}^{*}$ linear with respect to $F$ :

An operation $A$ assigning to every $\phi$ a definite corresponding $\phi^{*}=A(\phi)$ is called a homogeneous distributive operation of degree $n$ effective in a single valued way from $\mathfrak{M}$ to $\mathfrak{M}^{*}$ with respect to $F$, in case the $n+2$ elements

$$
\psi_{h}^{*}=A\left(\psi_{h}\right) \quad(h=0,1, \cdots, n+1)
$$

of $\mathfrak{M}^{*}$ corresponding to $n+2$ elements

$$
\psi_{h}=u_{h 1} \phi_{1}+u_{h_{2}} \phi_{2} \quad(h=0,1, \cdots, n+2)
$$

in a binary linear system $\left(\phi_{1}, \phi_{2}\right)$ in $\mathfrak{M}$ satisfy always the following relation :

$$
\left|\begin{array}{ccccc}
\psi_{0}^{*} & u_{01}^{n} & u_{01}^{n-1} u_{02} & \cdots & u_{02}^{n} \\
\cdot & \cdot & \cdot & \cdot & \\
\psi_{n+1}^{*} & u_{n+11}^{n} & u_{n+11}^{n-1} u_{n+12} & \cdots & u_{n+12}^{n}
\end{array}\right|=0 .
$$

It follows that there exist in an unique way single valued operations

$$
A_{\phi_{1}, \phi_{2}}^{n_{1}, n_{2}} \quad\left(n_{1}, n_{2}=0,1, \cdots, n ; n_{1}+n_{2}=n\right)
$$

on two arguments $\phi_{1}, \phi_{2}$ from $\mathfrak{M}$ to $\mathfrak{M}^{*}$ such that identically

$$
A\left(u_{1} \phi_{1}+u_{2} \phi_{2}\right)=\sum_{\substack{n_{1}, n_{2}=0 \\ n_{1}+n_{2}=n}}^{n} \frac{n !}{n_{1} ! n_{2} !} u_{1}^{n_{1}} u_{2}^{n_{2}} A_{\phi_{1}, \phi_{2}}^{n_{1}, n_{2}}
$$

Conversely, the existence of such operations with the identity $\left(B_{2}\right)$ implies that relation $\left(A_{2}\right)$ holds. Further similar identities $\left(A_{s}\right),\left(B_{s}\right)$ hold for every $s$-ary linear system $\left(\phi_{1}, \cdots, \phi_{s}\right)$.

The operations entering these identities are simply expressible in terms of the operation

$$
A_{\phi_{1}, \ldots, \phi_{n}}^{1, \ldots, 1}
$$

on $n$ arguments occurring in $\left(B_{n}\right)$; this operation is symmetric 
on its $n$ arguments and linear distributive on each argument, and

$$
A(\phi)=A_{\phi_{1}, \ldots, \phi_{n}}^{1, \ldots,}
$$

Conversely, if $A\left(\phi_{1}, \cdots, \phi_{n}\right)$ is an operation symmetric on its $n$ arguments and linear distributive on each, then

$$
A(\phi)=A(\phi, \cdots, \phi)
$$

is an operation on $\phi$ homogeneous distributive of degree $n$.

11. Recently Professor Ugo Amaldi has published in the Memorie of the Turin Academy a paper on the continuous groups of real conformal transformations in ordinary space. The groups were investigated by the methods of Lie and expressed in the usual infinitesimal notation. In Professor Newson's paper these real conformal transformations are studied by pure geometric methods and all types of such transformations are found. The subgroups of the real conformal group are determined and classified according to their types. Most of Professor Newson's results have been in his possession about eight years, but the investigation was completed only recently. The paper has been accepted for publication by the Giornale di Matematiche.

12. The first part of Professor Miller's paper is devoted to a proof of the theorem that a group is necessarily solvable if every non-abelian subgroup is dihedral. This theorem is then extended so as to read: if every non-abelian subgroup of a group is either dihedral or associate dihedral, the group is solvable. It is observed that every non-abelian subgroup of a dihedral group is dihedral, and all the non-dihedral groups in which every non-abelian subgroup is dihedral are investigated. It is proved that if such a group is non-abelian it must belong to one of the following five types: (1) the direct product of the octic group and a group of order $p, p$ being any prime number ; (2) the direct product of the dihedral group of order $2 q, q$ being an odd prime, and a group of order $p$; (3) the direct product of the dihedral group of order $4 q$ and a group of order $2 ;(4)$ the group obtained by extending the abelian group of order $p^{2}, p>2$, and of type $(1,1)$ by means of an operator of order 2 which transforms each operator of this abelian group into its inverse; (5) the group of order $4 q, q$ 
being prime and $\equiv 1 \bmod 4$, which is contained in the holomorph of the cyclic group of order $q$. The first three types contain invariant operators while there is no invariant operator besides identity in either of the last two types.

13. Professor Porter's first paper was a pedagogic note in which attention was called to the fact that if, for example, in three dimensions, we take as our element of volume

$$
\Delta=\frac{1}{3 !}\left|\begin{array}{lll}
\Delta_{1} x & \Delta_{2} x & \Delta_{3} x \\
\Delta_{1} y & \Delta_{2} y & \Delta_{3} y \\
\Delta_{1} z & \Delta_{2} z & \Delta_{3} z
\end{array}\right|
$$

and make the one to one change of variable $x=x(u, v, w)$, etc., we get

$$
\begin{aligned}
\Delta & =\frac{1}{3 !} J \cdot\left|\begin{array}{ccc}
\Delta_{1} u & \Delta_{2} u & \Delta_{3} u \\
\Delta_{1} v & \Delta_{2} v & \Delta_{3} v \\
\Delta_{1} w & \Delta_{2} w & \Delta_{3} w
\end{array}\right|(1+t) \\
& =\frac{1}{3 !} J \Delta^{\prime}(1+t),
\end{aligned}
$$

where $\lim _{\Delta^{\prime}=0} t=0$, provided the jacobian $J$ of the transformation is continuous.

If the jacobian does not change sign, the tetrahedron $\Delta^{\prime}$ will not overlap if the tetrahedra $\Delta$ do not by (1). The usual formulas for change of variable in triple integrals follow at once from Duhamel's principle.

14. Professor Porter's second paper contains further results of his research which began in the current number of the $A n$ nals of Mathematics and will be continued in the same journal.

15. The purpose of Dr. Wernicke's paper is to extend the applicability of Gauss's criterion for the interlocking of curves. In the double integral

$$
\frac{1}{4 \pi} \iint S \frac{\rho-\rho^{\prime}}{r^{3}} d \rho d \rho^{\prime}
$$

$S$ indicates the scalar product of the vectors, the curves $C, C^{\prime}$ being given by their vector equations $\rho=\rho(t), \rho^{\prime}=\rho^{\prime}(t)$; and 
$r$ is the length of $\rho-\rho^{\prime}$. This integral is an integer $m$ whenever $C$ coils $m$ times about $C^{\prime}$ in a fixed positive direction. Any bilateral simply connected surface bounded by $C^{\prime}$ is pierced at least $|m|$ times by $C$. It was shown that when $m=0, C$ may still so coil about $C^{\prime}$ and about itself that it cannot be "disentangled." A surface bounded by $C$ may be so constructed that $C^{\prime}$ does not pierce it. On such a surface, however, an auxiliary curve may be drawn so as to interlock with $C^{\prime}$ in the former way. Both ways of interlocking may occur simultaneously in the case both of the original and auxiliary curves, if the latter be properly chosen; but a repeated application of Gauss's criterion will reveal all these relations. Moreover, for $n$-space, the criterion may be so modified as to characterize the interlocking of closed $k$-surfaces with $(n-k-1)$-surfaces, where $k=0,1, \ldots, n-1$. Also, while the double integral is not immediately applicable to a curve interlocking with itself, or forming a " knot," it may be used to reveal knottedness, by extending it over the given curve and another accompanying it at a sufficiently small vectorial distance.

16. Dr. Westfall showed, by means of a recent theorem of Stekloff, that the fundamental theorem of Fourier's constants,

$$
\int_{a}^{b} A(x) f^{2}(x) d x=\sum_{i}^{\infty} a_{i}^{2}, \quad a_{i}=\int_{a}^{b} A(x) f(x) \phi_{i}(x) d x,
$$

holds for any limited and integrable function $f(x)$ and Hilbert's characteristic functions $\phi_{2}(x)$ of a self adjoined differential equation. It follows that, if $A(x)$ does not vanish in $(a, b)$, two limited and integrable functions having the same generalized Fourier's constants differ by an integrably null function in $(a, b)$, and if $\sum_{1}^{\infty} a_{i} \phi_{i}(x)$ converges to an integrable and finite function in any sub-interval $(a, \beta)$, or $(a, b)$, it likewise differs from $f(x)$ in that sub-interval by an integrally null function.

17. Professor Moore's note on the Fourier constants of an integrable function appears elsewhere in this number of the Bulletin.

18. Although linear transformations with a prime modulus $p$ are employed extensively in many branches of mathematics, the invariants of quantics under such transformations seem to have escaped attention. The first steps in the construction of a the- 
ory of such invariants are made in the paper by Professor Dickson. The subject is at once seen to be much more difficult than the ordinary algebraic theory. For instance, the terms of an invariant need not be of the same degree nor of constant weight. Again, the annihilators are far more complicated, involving partial derivatives of higher than the first order. Direct computation is necessarily a luxury in this field.

As the fundamental invariants of $a x^{2}+2 b x y+c y^{2}$, when $p>2$, we may take the discriminant $\Delta$ and

$$
J=\left(a^{\mu}+1\right)\left(c^{\mu}+1\right)\left\{\sum_{i=0}^{\mu} a^{i} c^{i} b^{2 \mu-2 i}-1\right\},
$$

where $\mu=\frac{1}{2}(p-1)$. Then $\Delta J=0$ and $(J+1)^{2}=I+1$, where

$$
I=\left(a^{p-1}-1\right)\left(b^{p-1}-1\right)\left(c^{p-1}-1\right) .
$$

Replacing $p$ by $p^{n}$, we obtain results valid for the Galois field of order $p^{n}$. Every quantic has an invariant analogous to $I$, viz., $\pi\left(a_{i}^{p^{n}-1}\right)$, the product extending over all the coefficients $a_{i}$ of the quantic.

A cubic form, for prime modulus $p>3$, has an absolute invariant $K$ of constant degree $p-1$ containing $2 m$ terms, where $m$ is the number of partitions of $p-1$ into $3 l_{0}+2 l_{1}+l_{2}$. Let $\Delta$ denote -3 times the algebraic discriminant $a_{0}^{2} \alpha_{3}^{2}+\cdots$ of the cubic form (with binomial coefficients). Then $\left(\Delta^{\frac{1}{2}(p+1)}-\Delta\right) K=0$.

As in these illustrations on quadratic and cubic forms, so in general the new invariants have simple relations with the ordinary algebraic invariants. These results will be incorporated in a paper offered to the Transactions.

19. Professor Pell showed that any curve of constant torsion may be considered as the locus of the middle points of the chords connecting corresponding points on a minimal curve and a spherical curve, correlated to this minimal curve. This theorem was obtained from Lie's formulas for constructing a minimal surface passing through a given contour, when the directions of the normals along this contour of the minimal surface are given.

20. For any power series $\sum_{0}^{\infty} a_{n} x^{n}$, with a radius of convergence $r$, such that both $\lim _{n=\infty} a_{n} x^{n}$ becomes definitely infinite and $\left|a_{n}^{-1} x^{-n} \sum_{0}^{n} a_{n} x^{n}\right|<\mathrm{L}$ while $|x|>r+e$, Professor 
Porter showed that there were points on the circle of convergence which are condensation points for the zeros of the polynomial convergents and that in the neighborhood of every point of this circle the set of these polynomials took on values less than any assigned number however small. He also showed that no set of these polynomials remained limited throughout any region lying outside the circle of convergence.

A. S. Chessin, Secretary.

\section{SELECTED TOPICS IN THE THEORY OF BOUND- ARY VALUE PROBLEMS OF DIFFER- ENTIAL EQUATIONS.}

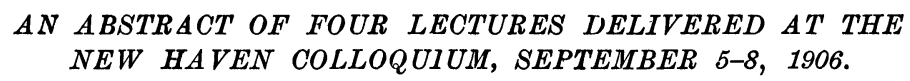

BY PROFESSOR MAX MASON.

\section{$\S 1$.}

Functional equations - particularly integral equations have aroused much interest and activity in recent years. Important contributions to the subject have been made, notably by Volterra, Fredholm and Hilbert, and the results have found application in the field of mathematical physics and differential equations. The equations studied have been for the most part of a type difficult of solution, and the treatment correspondingly complicated.

There is however a type of functional equation whose solution may be obtained in a simple manner. Consider the equation

$$
f=g+s f
$$

where $g$ is a known function, and $S$ a linear operator, that is $S(u+v)=S u+S v$. The operator $S$ will be called convergent if the infinite series

$$
\phi+S \phi+S^{2} \phi+S^{3} \phi+\cdots
$$

converges for all functions $\phi$ which satisfy the conditions of con- 